\title{
Analysis of Knowledge-sharing Evolutionary Game in University Teacher Team
}

\author{
Mingkui Huo ${ }^{1}$ \\ ${ }^{1}$ School of Economics and Management, Changchun University of Science and Technology, Changchun 130022, \\ China \\ Correspondence: Mingkui Huo, School of Economics and Management, Changchun University of Science and \\ Technology, Changchun 130022, China. E-mail: huomingkui@163.com
}

Received: January 17, 2013

Accepted: January 23, 2013

Online Published: January 24, 2013

doi:10.5430/ijhe.v2n1p60

URL: http://dx.doi.org/10.5430/ijhe.v2n1p60

\begin{abstract}
The knowledge-sharing activity is a major drive force behind the progress and innovation of university teacher team. Based on the evolutionary game theory, this article analyzes the knowledge-sharing process model of this team, studies the influencing mechanism of various factors such as knowledge aggregate gap, incentive coefficient and risk factor that influence the knowledge-sharing result, and accordingly proposes a strategy to promote the knowledge sharing of university teacher team.
\end{abstract}

Keywords: Teacher team, Knowledge sharing, Evolutionary game

\section{Introduction}

Teacher is the core resource promoting the development of universities, while the teacher team is the main supporter for teachers' existence and development. As an aggregate of new grass-roots organizations and core talents in the modern university system, the teacher team is a front responsible for fostering top-quality talents, that is, a teaching and research organization represented by discipline echelon, academic research center, task force, as well as teaching group. Members in this team may differ from each other in terms of capability, experience, skill, knowledge background, character and behavioral feature. But once making bold innovations through mutual complementation, mutual aid and mutual learning, they can achieve the team target smoothly while realizing their personal growth.

As a typical knowledge-based organization, the university teacher team will see the continuous interflow, exchange, formation, transfer and innovation of knowledge in its whole life, which, in nature, is just knowledge sharing. Knowledge sharing can not only improve the knowledge level of team members, but create a favorable learning climate in the team, consolidate the cooperation among members and lay a foundation for knowledge innovation. Hence the growth of teacher team is promoted.

\section{Literature review}

In recent years, the study on knowledge-sharing and knowledge management issues of university faculty has received the daily-increased attention of scholars and even yielded fruitful results.

Lynne proposed to achieve the synchronous and asynchronous knowledge exchange by means of electronic knowledge base (Lynne M. 2011). Hansen analyzed the knowledge-sharing conditions in the knowledge alliance (Hansen M T. 1999). Samaddar analyzed the resource-sharing problems like knowledge innovation in the cooperation course of two organizations (Samaddar S, Kadiyalas S. 2006). Zhou Dongsheng et. al recommended a knowledge management system based on J2EE framework and Web2.0 service (Zhou Dongsheng \& Zhao Yaping, 2011). Xu Ming established a five-dimensional model for university knowledge management from the perspectives of university knowledge, smallest faculty size, knowledge activities as well as knowledge management tool and environment (Qiao Wenzhong, 2008). Qiao Wenzhong, Guo Lan and Liao Fangwei insisted, the dissimilation level and channel and the reception capacity shackled the tacit knowledge interflow among university teachers (Luo HongYun, 2010). Through the principle-agent theory framework, they built a mathematical model for tacit knowledge transfer and sharing mechanism to optimize the strength of sharing mechanism (Wang Lili \& Jin XiuMing, 2009). Luo Hongyun, Lin Xiangyi and Gong Yanfen analyzed the key nodes and influencing factors of tacit knowledge sharing in the network environment (Wang Lei \& Wu DongHua, 2010). Wang Lili, Jin Xiuming, Lou Ying et al investigated and analyzed the drivers of knowledge sharing among university researchers, thus 
developing the knowledge-sharing strategies centered on spiritual, material and cultural incentives (Friedmand D. 1991).

Wang Lei and Wu Donghua proceeded with the teachers' individual intention to study in the questionnaire method the motives laying restraints on knowledge sharing behavior. With a structural equation model, Li Xia empirically analyzed the knowledge-sharing behavior of innovation teams in domestic 25 major universities. She suggested that for an innovative research team in a university, the resource sufficiency, cognitive conflicts, green hands, transformational leadership and membership heterogeneity would have a positive influence on their knowledge-sharing behavior, while the emotional conflicts would have a negative influence .

Thus it can be seen that in terms of knowledge sharing among university teachers, theoretical research is the mainstream and mostly follows such a main route as analyzing the sharing process, refining the influencing factors and proposing the strategy and mode. On the other hand, the research of influencing mechanism and the empirical study of knowledge-sharing activities are needy. Hence this article analyzed the process and relevant influencing factors of knowledge sharing among university teachers according to the evolutionary game theory, built a knowledge-sharing evolutionary game model, and analyzed the influencing mechanism of various factors. This is of certain theoretical and practical significance in enriching the university knowledge management theory and promoting the building and development of university teacher team.

\section{Knowledge-sharing evolutionary game model of university teacher team}

The evolutionary game theory has been developed on the basis of biology and classical game theory. It corrects the "rational economic man" hypothesis in the traditional game theory. Starting with individuals of bounded rationality, this theory has studied the group behavior and reasonably interpreted the evolutionary process of biological behaviors. It believes that like the evolution of life, any sub-optimal behavior will finally be eliminated, e.g. by competitive pressure and proper decision-making rules. With only bounded rationality, the gamer can't find an optimal equilibrium in every game, so his best strategy is to imitate and improve his and others' past best strategy. After a long time, all the gamers will incline to a stable strategy, which is called Evolutionary Stable strategy (ESS). The knowledge sharing activities among faculty members are quite compliant with evolutionary game feature under the bounded rationality.

To facilitate the building of knowledge-sharing evolutionary game model, this article studies the knowledge-sharing game between teacher team members $\mathrm{A}$ and $\mathrm{B}$ and makes the following hypotheses:

(1)The strategy set for both A and B is \{sharing knowledge and not sharing knowledge\}.

(2) If $Q_{A}$ and $Q_{B}$ are knowledge aggregates respectively owned by $\mathrm{A}$ and $\mathrm{B}$ and $\eta_{A}\left(0 \leq \eta_{A} \leq 1\right)$ and $\eta_{B}$ $\left(0 \leq \eta_{B} \leq 1\right)$ represent respectively their knowledge-sharing extent, then the amount of knowledge shared by both of them can be expressed by $Q_{A} \eta_{A}$ and $Q_{B} \eta_{B}$.

(3) Only the chiastopic fusion of shared or heterogeneous complementary knowledge is the original intention for team establishment. What is more, if what is shared is completely the overlapping knowledge, there will be no need to cooperate or share the knowledge. Therefore, if the proportion of complementary knowledge in the shared knowledge of members A and B is respectively expressed by $\mu_{A}\left(0 \leq \mu_{A} \leq 1\right)$ and $\mu_{B}\left(0 \leq \mu_{B} \leq 1\right)$, the value of shared meaningful knowledge that can be assimilated by the opposite side will be $Q_{A} \eta_{A} \mu_{A}$ and $Q_{B} \eta_{B} \mu_{B}$.

(4) In the face of same knowledge, different people can assimilate and convert a different amount of knowledge due to different personal circumstances. Therefore, if $\alpha_{A}\left(0 \leq \alpha_{A} \leq 1\right)$ and $\alpha_{B}\left(0 \leq \alpha_{B} \leq 1\right)$ represent respectively the knowledge assimilation capacity of members $A$ and $B$, then the value of knowledge obtained by the opposite side in a knowledge sharing activity will be respectively $Q_{B} \eta_{B} \mu_{B} \alpha_{A}$ and $Q_{A} \eta_{A} \mu_{A} \alpha_{B}$.

(5) In the knowledge sharing course, the synergetic and leverage effect of knowledge will help both parties A and B obtain more knowledge than $Q_{B} \eta_{B} \mu_{B} \alpha_{A}$ and $Q_{A} \eta_{A} \mu_{A} \alpha_{B}$ due to (e.g. in the brainstorming process, mutual intense discussion and rapid meditation will give birth to inspiration and subsequent new ideas and knowledge), which is called synergetic knowledge. If the size of synergetic knowledge is positively proportional to the knowledge amount shared by the opposite side and the knowledge synergy coefficient of $\mathrm{A}$ and $\mathrm{B}$ is respectively $\beta_{A}$ and $\beta_{B}$, then on condition that both A and B have spontaneously chosen the knowledge-sharing strategy, the newly increased value of synergetic knowledge for both people will be $Q_{B} \eta_{B} \mu_{B} \beta_{A}$ and $Q_{A} \eta_{A} \mu_{A} \beta_{B}$ and their added knowledge value on the original basis shall be $Q_{B} \eta_{B} \mu_{B} \alpha_{A}+Q_{B} \eta_{B} \mu_{B} \beta_{A}$ and $Q_{A} \eta_{A} \mu_{A} \alpha_{B}+Q_{B} \eta_{B} \mu_{B} \beta_{A}$. 
(6) As a whole, when the team has achieved the target or made tremendous progress, the team members ought to be rewarded and those with more contributions shall deserve a greater reward. This reward, to some extent, can be converted into a knowledge-sharing incentive. Therefore, suppose the knowledge-sharing incentive coefficient is $\lambda$, the received incentive value of $\mathrm{A}$ and $\mathrm{B}$ based on their shared knowledge amount will be respectively $\lambda Q_{A} \eta_{A} \mu_{A}$ and $\lambda Q_{B} \eta_{B} \mu_{B}$.

(7) In the knowledge-sharing course, another negative phenomenon may loom large. When one side is aware that his received knowledge value is much less than his contributed knowledge value and the case for the opposite site is just the reverse, the former's knowledge advantage will gradually be smoothed out. On condition that the former's position and interests based on his knowledge advantage have suffered a heavy loss, the former may reduce and even refuse the knowledge sharing. This means the knowledge-sharing activity itself is of some risk and normally the party with higher knowledge advantage bears a greater risk. Therefore, if $r_{A}$ and $r_{B}$ express respectively the risk coefficient of members $\mathrm{A}$ and $\mathrm{B}$ in adopting the knowledge-sharing strategy, the subsequent knowledge loss incurred by both members shall be $r_{A} Q_{A} \eta_{A}$ and $r_{B} Q_{B} \eta_{B}$. In addition, as the tacit knowledge is hard to describe and measure, the shared knowledge can't be effectively quantified so that the loss incurred by the members may be far more than their received knowledge-sharing incentive. Thus this test assumes $r_{A} Q_{A} \eta_{A}>\lambda Q_{A} \eta_{A} \mu_{A}$ and $r_{B} Q_{B} \eta_{B}>\lambda Q_{B} \eta_{B} \mu_{B}$

(8) When both A and B choose the non-sharing strategy, they will have no knowledge increment. As a result, both of them will claim zero gains.

According to the above analysis, a matrix of knowledge-sharing game payoff between faculty members A and B can be prepared as in Table 1.

Table 1. Knowledge-sharing game payoff matrix of university teacher team

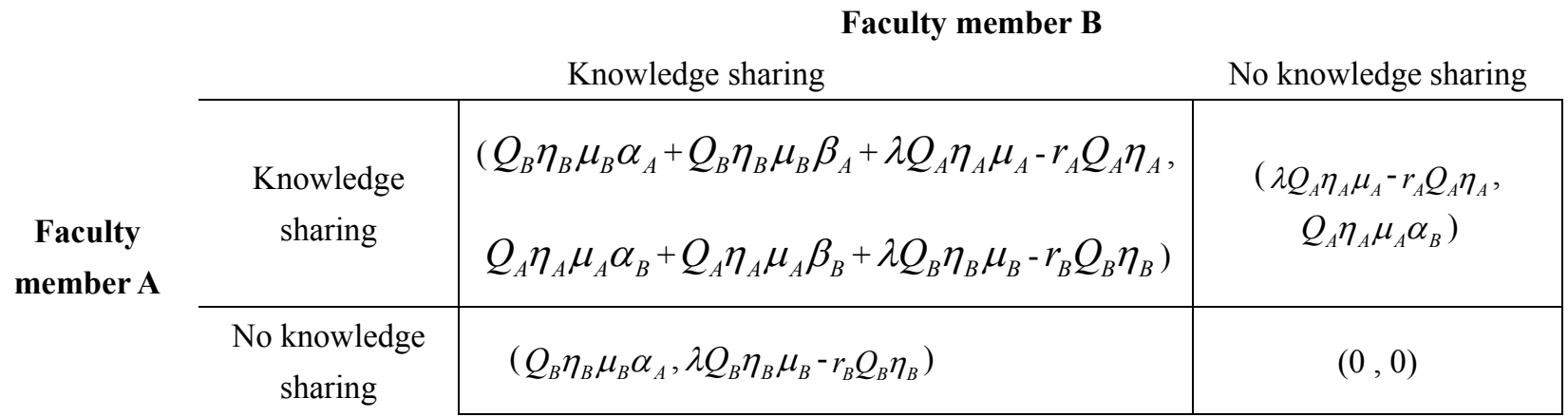

\section{Analysis of equilibrium points on the knowledge-sharing game payoff model of university teacher team}

Suppose the knowledge-sharing probability of member $\mathrm{A}$ is $x$ and his non-sharing probability is $1-x$, and the knowledge-sharing probability of member $\mathrm{B}$ is $y$ and his non-sharing probability is $1-y$. If $E_{A 1}$ and $E_{B 1}$ are the revenue of $\mathrm{A}$ and $\mathrm{B}$ in choosing the knowledge sharing strategy, $E_{A 2}$ and $E_{B 2}$ are the revenue of $\mathrm{A}$ and $\mathrm{B}$ in choosing the non-sharing strategy, and $\overline{E_{A}}$ and $\overline{E_{B}}$ are average revenue of $\mathrm{A}$ and $\mathrm{B}$, then we can determine:

$$
\begin{aligned}
& E_{A 1}=y\left(Q_{B} \eta_{B} \mu_{B} \alpha_{A}+Q_{B} \eta_{B} \mu_{B} \beta_{A}+\lambda Q_{A} \eta_{A} \mu_{A}-r_{A} Q_{A} \eta_{A}\right)+(1-y)\left(\lambda Q_{A} \eta_{A} \mu_{A}-r_{A} Q_{A} \eta_{A}\right) \\
& =y Q_{B} \eta_{B} \mu_{B} \alpha_{A}+y Q_{B} \eta_{B} \mu_{B} \beta_{A}+\lambda Q_{A} \eta_{A} \mu_{A}-r_{A} Q_{A} \eta_{A} \text {; } \\
& E_{A 2}=y Q_{B} \eta_{B} \mu_{B} \alpha_{A} \text {; } \\
& \overline{E_{A}}=x E_{A 1}+(1-x) E_{A 2}=y Q_{B} \eta_{B} \mu_{B} \alpha_{A}+x y Q_{B} \eta_{B} \mu_{B} \beta_{A}+x \lambda Q_{A} \eta_{A} \mu_{A}-x r_{A} Q_{A} \eta_{A} \\
& E_{B 1}=x\left(Q_{A} \eta_{A} \mu_{A} \alpha_{B}+Q_{A} \eta_{A} \mu_{A} \beta_{B}+\lambda Q_{B} \eta_{B} \mu_{B}-r_{B} Q_{B} \eta_{B}\right)+(1-x)\left(\lambda Q_{B} \eta_{B} \mu_{B}-r_{B} Q_{B} \eta_{B}\right) \\
& =x Q_{A} S_{A} \mu_{A} \alpha_{B}+x Q_{A} \eta_{A} \mu_{A} \beta_{B}+\lambda Q_{B} \eta_{B} \mu_{B}-r_{B} Q_{B} \eta_{B} \\
& E_{B 2}=x Q_{A} \eta_{A} \mu_{A} \alpha_{B} \\
& \overline{E_{B}}=y E_{B 1}+(1-y) E_{B 2}=x Q_{A} \eta_{A} \mu_{A} \alpha_{B}+x y Q_{A} \eta_{A} \mu_{A} \beta_{B}+y \lambda Q_{B} \eta_{B} \mu_{B}-y r_{B} Q_{B} \eta_{B}
\end{aligned}
$$


Next, the replicated dynamic equation of members A and B can be determined as:

$$
\begin{aligned}
& F(x)=\frac{d_{x}}{d_{t}}=x\left(E_{A 1}-\overline{E_{A}}\right)=x(1-x)\left(y Q_{B} \eta_{B} \mu_{B} \beta_{A}+\lambda Q_{A} \eta_{A} \mu_{A}-r_{A} Q_{A} \eta_{A}\right) \\
& F(y)=\frac{d_{y}}{d_{t}}=y\left(E_{B 1}-\overline{E_{B}}\right)=y(1-y)\left(x Q_{A} \eta_{A} \mu_{A} \beta_{B}+\lambda Q_{B} \eta_{B} \mu_{B}-r_{B} Q_{B} \eta_{B}\right)
\end{aligned}
$$

The system of differential equations consisting of equations (7) and (8) can describe the knowledge-sharing game evolution process of university teacher team. By analyzing the equilibrium stability of evolution system through the local stability of Jacobian matrix ${ }^{[16]}$, the equilibrium points $(x, y)$ of this system can be determined as: $\mathrm{D}_{1}(0,0)$, $\mathrm{D}_{2}(1,0), \mathrm{D}_{3}(1,1), \mathrm{D}_{4}(0,1), \mathrm{D}_{5}\left(\frac{r_{A} Q_{A} \eta_{A}-\lambda Q_{A} \eta_{A} \mu_{A}}{Q_{B} \eta_{B} \mu_{B} \beta_{A}}\right.$ and $\left.\frac{r_{B} Q_{B} \eta_{B}-\lambda Q_{B} \eta_{B} \mu_{B}}{Q_{A} \eta_{A} \mu_{A} \beta_{B}}\right) ;$

By determining the partial derivative of $\mathrm{x}$ and $\mathrm{y}$ in the above two equations, the Jacobian matrix can be deduced:

$$
J=\left[\begin{array}{l}
\frac{\partial F(x)}{\partial x}, \frac{\partial F(x)}{\partial y} \\
\frac{\partial F(y)}{\partial x}, \frac{\partial F(y)}{\partial y}
\end{array}\right]=\left[\begin{array}{l}
\left.(1-2 x)\left(y Q_{B} \eta_{B} \mu_{B} \beta_{A}+\lambda Q_{A} \eta_{A} \mu_{A}-r_{A} Q_{A} \eta_{A}\right), x(1-x) Q_{B} \eta_{B} \mu_{B} \beta_{A}\right) \\
y(1-y) Q_{A} \eta_{A} \mu_{A} \beta_{B},(1-2 y)\left(x Q_{A} \eta_{A} \mu_{A} \beta_{B}+\lambda Q_{B} \eta_{B} \mu_{B}-r_{B} Q_{B} \eta_{B}\right)
\end{array}\right]
$$

According to two requirements, namely whether the Jacobian matrix on the above five equilibrium points complies with $|J|>0$ and the value of trace is $\operatorname{tr}(J)<0$, the existence of local stability of equilibrium points can be judged. Among these points, the points $\mathrm{D}_{1}$ and $\mathrm{D}_{3}$ are locally stable within the evolutionary stable strategy (ESS), but

\begin{tabular}{|c|c|c|c|}
\hline $\begin{array}{l}\text { Equilibrium point } \\
(x, y)\end{array}$ & Value of $|J|$ & Value of $\operatorname{tr}(J)$ & $\begin{array}{l}\text { Local } \\
\text { stability }\end{array}$ \\
\hline $\mathbf{D}_{1} \quad(0,0)$ & $\begin{array}{l}\left(\lambda Q_{A} \eta_{A} \mu_{A}-r_{A} Q_{A} \eta_{A}\right) \\
\left(\lambda Q_{B} \eta_{B} \mu_{B}-r_{B} Q_{B} \eta_{B}\right) \\
>_{0}\end{array}$ & $\begin{array}{l}\left(\lambda Q_{A} \eta_{A} \mu_{A}-r_{A} Q_{A} \eta_{A}\right)+ \\
\left(\lambda Q_{B} \eta_{B} \mu_{B}-r_{B} Q_{B} \eta_{B}\right) \\
<0\end{array}$ & $\begin{array}{l}\text { Stable } \\
\text { point }\end{array}$ \\
\hline $\mathbf{D}_{2} \quad(1,0)$ & $\begin{array}{l}\left(r_{A} Q_{A} \eta_{A}-\lambda Q_{A} \eta_{A} \mu_{A}\right) . \\
\left(\lambda Q_{B} \eta_{B} \mu_{B}+Q_{A} \eta_{A} \mu_{A} \beta_{B}-r_{B} Q_{B} \eta_{B}\right) \\
>0\end{array}$ & $\begin{array}{l}\left(r_{A} Q_{A} \eta_{A}-\lambda Q_{A} \eta_{A} \mu_{A}\right)+ \\
\left(\lambda Q_{B} \eta_{B} \mu_{B}+Q_{A} \eta_{A} \mu_{A} \beta_{B}-r_{B} Q_{B} \eta_{B}\right) \\
>0\end{array}$ & $\begin{array}{l}\text { Unstable } \\
\text { point }\end{array}$ \\
\hline $\mathbf{D}_{\mathbf{3}} \quad(1,1)$ & $\begin{array}{l}\left(Q_{B} \eta_{B} \mu_{B} \beta_{A}+\lambda Q_{A} \eta_{A} \mu_{A}-r_{A} Q_{A} \eta_{A}\right) \\
\left(Q_{A} \eta_{A} \mu_{A} \beta_{B}+\lambda Q_{B} \eta_{B} \mu_{B}-r_{B} Q_{B} \eta_{B}\right) \\
>0\end{array}$ & $\begin{array}{l}(-1)\left(Q_{B} \eta_{B} \mu_{B} \beta_{A}+\lambda Q_{A} \eta_{A} \mu_{A}-r_{A} Q_{A} \eta_{A}\right)+<0 \\
(-1)\left(Q_{A} \eta_{A} \mu_{A} \beta_{B}+\lambda Q_{B} \eta_{B} \mu_{B}-r_{B} Q_{B} \eta_{B}\right)\end{array}$ & $\begin{array}{l}\text { Stable } \\
\text { point }\end{array}$ \\
\hline $\mathbf{D}_{4} \quad(0,1)$ & $\begin{array}{l}\left(Q_{B} \eta_{B} \mu_{B} \beta_{A}+\lambda Q_{A} \eta_{A} \mu_{A}-r_{A} Q_{A} \eta_{A}\right) . \\
(-1)\left(\lambda Q_{B} \eta_{B} \mu_{B}-r_{B} Q_{B} \eta_{B}\right) \\
>0\end{array}$ & $\begin{array}{l}\left(Q_{B} \eta_{B} \mu_{B} \beta_{A}+\lambda Q_{A} \eta_{A} \mu_{A}-r_{A} Q_{A} \eta_{A}\right) \\
-\left(\lambda Q_{B} \eta_{B} \mu_{B}-r_{B} Q_{B} \eta_{B}\right) \\
>0\end{array}$ & $\begin{array}{l}\text { Unstable } \\
\text { point }\end{array}$ \\
\hline $\begin{array}{l}\mathbf{D}_{\mathbf{5}}( \\
\frac{r_{A} Q_{A} \eta_{A}-\lambda Q_{A} \eta_{A} \mu_{A}}{Q_{B} \eta_{B} \mu_{B} \beta_{A}} \\
\left.\frac{r_{B} Q_{B} \eta_{B}-\lambda Q_{B} \eta_{B} \mu_{B}}{Q_{A} \eta_{A} \mu_{A} \beta_{B}}\right)\end{array}$ & $\begin{array}{l}\frac{-\left(r_{A} Q_{A} \eta_{A}-\lambda Q_{A} \eta_{A} \mu_{A}\right) \cdot\left(Q_{B} \eta_{B} \mu_{B} \beta_{A}-r_{A} Q_{A} \eta_{A}+\lambda Q_{A} \eta_{A} \mu_{A}\right)}{Q_{B} \eta_{B} \mu_{B} \beta_{A}} \\
\frac{\left(r_{B} Q_{B} \eta_{B}-\lambda Q_{B} \eta_{B} \mu_{B}\right)\left(Q_{A} \eta_{A} \mu_{A} \beta_{B}-r_{B} Q_{B} \eta_{B}+\lambda Q_{B} \eta_{B} \mu_{B}\right)}{Q_{A} \eta_{A} \mu_{A} \beta_{B}} \\
<0\end{array}$ & $=0$ & $\begin{array}{l}\text { Saddle } \\
\text { point }\end{array}$ \\
\hline
\end{tabular}
the points $\mathrm{D}_{2}$ and $\mathrm{D}_{4}$ are unstable and the point $\mathrm{D}_{5}$ is a saddle point. For the detailed analysis, see Table 2:

Table 2. equilibrium points in the knowledge-sharing evolutionary game of university teacher team 


\section{Idea of knowledge-sharing promotion strategies for teacher team}

The evolution phase diagram of this game system can be drawn according to Table 2 (see Fig. 1).

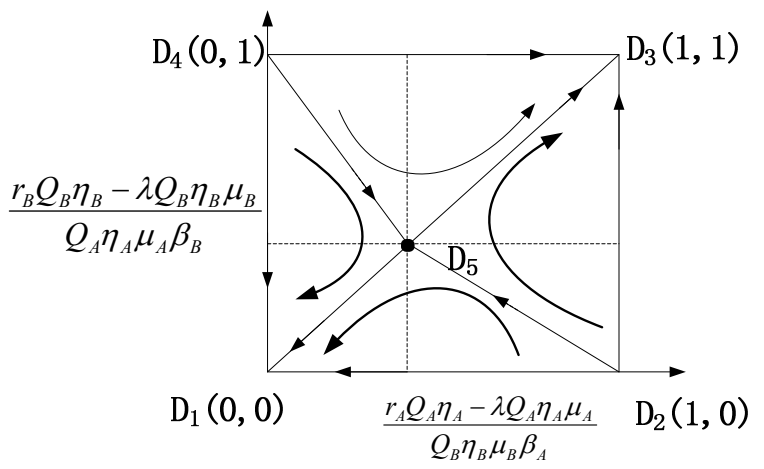

Figure 1. Knowledge-sharing game evolution phases of team members A and B

As shown in this diagram, the knowledge-sharing game of team members $A$ and $B$ converges to point $\mathrm{D}_{1}$ or $\mathrm{D}_{3}$, that is, the final equilibrium solution of this game evolution system is either (knowledge sharing, knowledge sharing) or (no knowledge sharing, no knowledge sharing). The $\mathrm{S}_{1}$ and $\mathrm{S}_{2}$, namely the area of figures $\mathrm{D}_{1} \mathrm{D}_{2} \mathrm{D}_{5} \mathrm{D}_{4}$ and $\mathrm{D}_{2} \mathrm{D}_{5} \mathrm{D}_{4} \mathrm{D}_{3}$, represent the probability of game convergence to points $\mathrm{D}_{1}$ or $\mathrm{D}_{3}$ respectively. Hence the knowledge-sharing promotion strategies for university teacher team lie in how to increase the $S_{2}$ and decrease the $S_{1}$.

$$
S_{1}=\frac{1}{2}\left(\frac{r_{B} Q_{B} \eta_{B}-\lambda Q_{B} \eta_{B} \mu_{B}}{Q_{A} \eta_{A} \mu_{A} \beta_{B}}+\frac{r_{A} Q_{A} \eta_{A}-\lambda Q_{A} \eta_{A} \mu_{A}}{Q_{B} \eta_{B} \mu_{B} \beta_{A}}\right)
$$

$S_{2}=1-S_{1}$

Analysis of equations (9) and (10:

(1)When the knowledge aggregates for members A and $\mathrm{B}$, namely $Q_{A}$ and $Q_{B}$, change proportionally but other variables remain unchanged, the value of $\mathrm{S}_{1}$ and $\mathrm{S}_{2}$ won't change. When the knowledge aggregate of one side is much bigger than that of the opposite side, e.g. $Q_{A} \gg Q_{B}$ or $Q_{B} \gg Q_{A}$, the value of $\mathrm{S}_{2}$ will approach zero and then the system will converge to point $D_{1}$. But when the value of $S_{1}$ is close to that of $S_{2}$, the value of $S_{1}$ will approach zero and then the system will converge to point $\mathrm{D}_{3}$.

(2)When the knowledge-sharing extents $\eta_{A}$ and $\eta_{B}$ get larger, the value of $\mathrm{S}_{1}$ will decrease while the value of $\mathrm{S}_{2}$ will increase. Consequently, the probability of system converging to point $\mathrm{D}_{3}$ will be higher, resulting in a greater chance of knowledge-sharing strategy implementation by both sides.

(3)When the proportions of complementary knowledge $\mu_{A}$ and $\mu_{B}$ get higher, the value of $\mathrm{S}_{1}$ will decrease while the value of $S_{2}$ will increase. Consequently, the probability of system converging to point $\mathrm{D}_{3}$ will be higher, resulting in a greater chance of knowledge-sharing strategy implementation by both sides.

(4)When the knowledge-sharing incentive coefficient $\lambda$ gets bigger, the value of $S_{1}$ will decrease while the value of $\mathrm{S}_{2}$ will increase, so that the probability of system converging to point $\mathrm{D}_{3}$ will be higher. This means when the team can evaluate the knowledge-sharing effect scientifically and objectively and develop a high-standard incentive scheme, the probability that all the members spontaneously choose the knowledge-sharing strategy will become higher.

(5)When the knowledge-sharing risk factors $r_{A}$ and $r_{B}$ get bigger, the value of $\mathrm{S}_{1}$ will increase while the value of $S_{2}$ will decrease, so that the probability of system converging to point $D_{1}$ will be higher.

(6)In the knowledge-sharing course, the increase in synergy coefficients $\beta_{A}$ and $\beta_{B}$ will lead to the decrease in $S_{1}$ and the increase in $S_{2}$ and the consequent higher probability of system converging to point $D_{3}$.

Therefore, based on the above analysis, this article has proposed the following strategies to promote the knowledge sharing among university teaching members.

Strategy 1: In establishing a teacher team, it shall be avoided by all means to choose those members differing greatly in knowledge and capability. The gap in teachers' experience, technical title and education can be utilized to build an 
echelon-like team, such as a teacher team comprising professors, associate professors, lecturers and assistants in a proportional drop structure. This can avoid not only the loss of motivation of high-level members due to unilateral guidance but the "hitch-hike" or blind learning of low-level members.

Strategy 2: Establish a clear teaming roadmap, clarify the task target, intensify the teamwork awareness, provide a convenient information technology environment, and create a team climate featuring mutual trust, mutual support and mutual aid. In this way, the team-based knowledge-sharing willingness can be enhanced to expand the knowledge shared by members.

Strategy 3: In choosing the university teaming members, strong attention to the gap in knowledge background, discipline, major and even research direction as well as strengthened multi-discipline, multi-major and multi-unit crossover study can help the members learn from each other and optimize their knowledge-sharing result.

Strategy 4: Develop a fair, rational performance evaluation system. Lay stress on the effective combination of hygienic factor and incentive factor. Proceed from the members' actual needs to establish a perfect team incentive system whose core is how to correctly measure the "quantity" and "quality" of shared knowledge.

Strategy 5: The knowledge sharing is risky, in particular for those members whose knowledge aggregate is in a leading position. They have more concerns about knowledge sharing, so a knowledge protection system and a knowledge-related loss compensation system must be established to soothe their concerns. For example, there shall be a rational supervision mechanism to guard against the divulgence of knowledge, some effective punitive measures to restrain the hitchhike and opportunistic behaviors, and an internal copyright system to settle the knowledge utilization difference.

Strategy 6: The value of synergy coefficients $\beta_{A}$ and $\beta_{B}$ is obviously objective, as it varies with not only environment, conditions and atmosphere but human character and knowledge background. In spite of the difficult in accurately quantifying this value, this article believes the following measures have played a certain active role. Firstly, create an office climate favorable to the exchange and interaction of knowledge. Secondly, make active use of the interaction of various complementary information technologies such as program and mailing list. Thirdly, choose a correct method to gather the information, and create a heated discussion atmosphere such as brainstorm and round-table conference. Fourthly, choose the members that are complementary in terms of character and manner. Especially when selecting the team leader, in addition to capability, the factors such as personal prestige and interpersonal relationship shall also be comprehensively considered. Fifthly, give full play to the role of core members. Though the knowledge sharing of faculty is mainly centered on core members, this doesn't mean the non-core members only play a role of pure knowledge assimilator. The knowledge shared by non-core members is also of significance in arousing the knowledge innovation and inspiration of core members.

\section{Conclusions}

The university teacher team is a front fostering the top-quality talents, and is a core unit realizing the technical and knowledge innovation of a university. The teacher team is established to achieve the " $1+1>2$ " result as well as the complementarities and mutual aid for teachers to jointly complete various tasks. Sharing the knowledge is a core faculty activity, but is not in smooth sailing. Considering the unbalance of knowledge-sharing cost and revenue and the necessity to maintain their position, some core members may intentionally "hide their weak points". Meanwhile, some members may adopt the "hitchhike" and opportunistic behaviors, which can remarkably weaken the knowledge-sharing willingness of a whole team, thus triggering the vicious cycles. This article believes that whether the faculty members will share the knowledge depends on the result of behavioral game among members. By referring to the evolutionary game theory, the article studies the knowledge-sharing process of teacher team, builds a knowledge-sharing evolutionary game model, analyzes the influencing mechanism of various factors such as member knowledge gap, knowledge-sharing incentive coefficient and knowledge-sharing risk factor, and accordingly presents the corresponding solutions.

Even so, as the faculty activities in real life tend to be more complicated, the factors influencing the knowledge sharing tend to increase. In addition, more in-depth research and even demonstration are to be made for some quantification problems proposed in this article, for example, how to accurately and objectively weigh the members' contributions to knowledge sharing and knowledge innovation and how to develop a fair and rational incentive system. 


\section{References}

Friedmand D. (1991). Evolutionary games in economics. Econometrica, 1991(3): 637-666. http://dx.doi.org/10.2307/2938222

Hansen M T. (1999). The search-transfer problem: the role of weak ties in sharing knowledge across organization subunits. Administrative Science Quarterly, 1999(1):82-111. http://dx.doi.org/10.2307/2667032

Luo, HongYun. (2010). Analysis of influence factors of university teachers' tacit knowledge sharing in Network environment. Journal of information, 2010(3): 116-119.

Lynne M. (2001). Toward a Theory of Knowledge Reuse Types of Knowledge Reuse Situations and Factors in Reuse Success. Journal of Management Information System, 2001(1):57-93.

Qiao, Wenzhong. (2008). Of university teachers' tacit knowledge sharing difficulties. Information theory and practice, 2008(5): 659-662.

Samaddar S, Kadiyalas S. (2006). An analysis of interorganizational resource sharing decisions in collaborative knowledge creation. European Journal of Operational Research, 2006(1): 192-210. http://dx.doi.org/10.1016/j.ejor.2004.06.024

Wang, Lei \& Wu, DongHua. (2010). The research of University teachers' knowledge sharing motivation and behavior. library science research, 2010(2): 94-97.

Wang, Lili \& Jin, XiuMing. (2009). Study on knowledge sharing mechanism and strategy of Scientific research team. Journal of Wuhan University of Engineering, 2009(4): 56-59.

Zhou, Dongsheng \& Zhao, Yaping. (2011). Based on the knowledge sharing of university research team knowledge management system. Experiment technology and Management, 2011(4): 250-252, 255. 